ARTÍCULO ORIGINAL

\title{
Caracterización de la enfermedad de la artritis y encefalitis caprina en las provincias de Salta y Jujuy
}

\author{
Dodero $\mathrm{AM}^{1^{*}}$, Micheloud $\mathrm{JF}^{1}$, Alfaro $\mathrm{JR}^{2}$, Alfaro $\mathrm{EJ}^{2}$, Pinto $\mathrm{GB}^{3}$, Suarez $\mathrm{VH}^{1}$ \\ ${ }^{1}$ INTA - AISA-IIACS Salta, Instituto de Investigación Animal del Chaco Semiárido \\ ${ }^{2}$ INTA - Estación Experimental Salta \\ ${ }^{3}$ INTA - Instituto de Virología-CICVyA, Hurlingham, Buenos Aires.
}

* Correspondencia: Ana M. Dodero, INTA Salta, RN 68, km 172, Cerrillos, 4403, Salta, Argentina. E-mail: dodero.ana@inta.gob.ar

Recibido: 24 Febrero 2017. Aceptado: 5 Junio 2017. Disponible en línea: 6 Junio 2017

Editor: P.M. Beldomenico

RESUMEN. La artritis y encefalitis Caprina (CAEV) puede presentar diferentes síntomas clínicos, siendo la artritis la más común. A fin de caracterizar la presencia de enfermedades en las majadas caprinas del noroeste argentino, se llevó a cabo una encuesta sanitaria a productores, con toma de muestras biológicas para el diagnóstico de enfermedades en la que fue incluida CAEV. Se efectuó un análisis serológico con un ELISA indirecto y se completó con un examen clínico general de los animales y toma de muestra de necropsia de algunos de los casos. En la Provincia de Salta, tres establecimientos fueron positivos, con un 6,25\% de prevalencia. La seroprevalencia del hato con seguimiento de caso fue del 55\% mientras que el $48 \%$ presentó signos clínicos donde la artritis de la articulación carpal fue la más frecuentemente afectada. El virus CAEV está distribuido en la provincia de Salta y la severidad de las lesiones observadas sugiere la presencia de nuevas cepas que deben ser investigadas conjuntamente al desarrollo de capacidades locales para el diagnóstico, clave de cualquier plan de erradicación.

SUMMARY. Characterization of caprine arthritis-encephalitis disease in Salta and Jujuy provinces. Caprine arthritis and encephalitis virus (CAEV) may present different clinical symptoms, with arthritis being the most common. In order to characterize the presence of diseases in goats from the Argentinean northwest, a sanitary survey was carried out to producers, with biological sampling for the diagnosis of diseases in which CAEV was included. A serological analysis was performed with an indirect ELISA and was completed with a general clinical examination of the animals and a necropsy sample collection of some of the cases. In the Province of Salta, three establishments were positive, with a prevalence of $6.25 \%$. The serum prevalence of the herd with case follow-up was $55 \%$, while $48 \%$ presented clinical signs where arthritis of the carpal joint was most frequently affected. The CAEV virus is spread in the province of Salta and the severity of the observed lesions suggests the presence of new strains that should be investigated in combination with the development of local capacities for diagnosis, this is the key to any eradication plan.

Palabras clave: Retrovirus; cabras; artritis; Salta; Jujuy.

Key words: Retroviruses, goats, arthritis, Salta, Jujuy.

\section{Introducción}

Los lentivirus de los pequeños rumiantes (SRLV) incluyen a los virus de la Artritis-Encefalitis Caprina y al Maedi-Visna, que pertenecen a la familia Retroviridae, subfamilia Orthoretrovirinae, género lentivirus. Afectan a ovinos y caprinos transmitiéndose naturalmente entre ambas especies (Narayan et al, 1980; Pepin et al, 1998; Jolly et al, 1989; Minguijon et al, 2015) y pueden producir cuatro formas clínicas clásicas: pulmonar, nerviosa, mamaria y articular. La forma pulmonar se caracteriza histopatológicamente por neumonía del tipo intersticial, notorio incremento de tamaño visceral, acompañado de disnea y caquexia; la forma nerviosa se produce en animales jóvenes, entre 2 y 3 meses presentando una encefalitis linfocítica con o sin afección medular y los animales se muestran atáxicos; en la glándula mamaria se produce una mastitis intersticial con fibrosis, reducción de la producción láctea y el endurecimiento del tejido; por último la forma articular se caracteriza por una proliferación de la membrana sinovial en capas que causa un engrosamiento artrítico con erosión de las superficies articulares, principalmente carpos, que produce rengueras crónicas, menor consumo, producción e incluso postración del animal (Narayan et al, 1980; Minguijon et al, 2015; De Souza, 2015; Zink y Jonhnson, 1994). La artritis crónica se observa principalmente en animales mayores de un año (Jones 
et al, 1997; Smith y Sherman, 1994; Radostits et al, 2002).

La principal forma de transmisión de la enfermedad es la horizontal, a través de la leche o el calostro de cabras infectadas (Rowe et al, 1997), además por secreciones vaginales, sangre, saliva o secreciones respiratorias; está demostrada la trasmisión seminal, incluso por inseminación artificial y en forma experimental se ha reproducido la vía transmamaria. La infección intrauterina, la transmisión iatrogénica y por fómites también tienen un rol importante en la trasmisión de la enfermedad (Rowe et al, 1997, Lujan et al, 2001, Minguijon et al, 2015).

El virus se detectó en la Argentina serológicamente por primera vez en ovinos en 2001 en la provincia de Río Negro (Robles et al, 2003) y a partir de entonces se lo ha encontrado con prevalencias crecientes en casi todo el país, y en San Luis a finales de 2015 (Rossanigo et al, 2016a y 2016b; Álvarez et al, 2016) y el referido en el presente trabajo (Dodero et al, 2016; Zabal et al, 2016) - reportado ante SENASA en Salta durante el año 2016 - contribuyendo al cambio del estatus sanitario del país comunicado en septiembre de 2016 por SENASA en su boletín oficial junto a la nueva reglamentación para transporte y comercialización de reproductores mayores de 6 meses de edad (https://www.boletinoficial.gob.ar/\#!DetalleNorma/15 1302/20160923 27/10/2016). El primer reporte de un aislamiento en el país, data de febrero de 2017 (Panei et al., 2017). La cepa aislada en la provincia de Buenos Aires corresponde al genotipo B, subtipo B1, con un porcentaje de homología del $86-92 \%$ con las estirpes anteriormente publicadas por Cork 1974.

Se propuso mostrar la situación epidemiológica de la Artritis encefalitis caprina en las provincias de Salta y Jujuy a través de las seroprevalencias obtenidas entre los años 2011-2016 haciendo la descripción correspondiente a los datos obtenidos de una encuesta sanitaria realizada a productores caprineros en la que se tomaron de muestras biológicas para el diagnóstico de diferentes enfermedades en la que fue incluida CAEV (Dodero et al, 2016). Al encontrarse resultados positivos y con la posibilidad de trabajar en el seguimiento de un caso natural se incorporó información generada a partir de ese brote denunciado.

\section{Materiales y Métodos}

Entre abril y noviembre de 2011 a 2016 se realizó una encuesta ad hoc a pequeños productores familiares de caprinos que a través de preguntas y con la ayuda de fotos de lesiones, se recabaron datos sanitarios, de manejo general y productivo en 78 establecimientos. De cada uno se tomaron muestras de suero de entre 20 y 25 hembras adultas. Las ecorregiones estudiadas fueron: Quebradas Áridas (QA), incluyendo la quebra- da de Humahuaca y la quebrada del Río Toro, el Chaco Salteño (CS), Departamentos de Anta y Rivadavia y Valles Templados (VT), en este último caso el Valle de Lerma.

Se procesaron en total 1819 muestras de sueros para el diagnóstico de diferentes patologías y se realizó la prueba de ELISA IDEXX CAEV/MVV Total Ab (Checkit), según las recomendaciones del fabricante para detectar anticuerpos específicos contra CAEV. Mientras que la prueba IDEXX MVV/CAEV p28 Ab Verification fue utilizada como método confirmatorio. De esta forma se detectó la circulación viral en la provincia de Salta (Dodero et al, 2015).

Con posterioridad al diagnóstico serológico positivo, se realizó el seguimiento de un establecimiento. Dicho hato se ubicó en la ecorregión de VT. Contaba con características tales como: accesibilidad, predisposición al trabajo e identificación individual de todos los animales y presencia de signos clínicos compatibles con esta enfermedad. Se sangraron 111 animales adultos de los cuales 20 fueron evaluados con Checkit. Se realizó una revisación clínica exhaustiva total con énfasis en aparato locomotor, acompañado de un seguimiento clínico durante todo el año con el sacrificio y toma de muestras de algunos de los casos.

Se siguió el protocolo de necropsia descrito por Robles (1991), y la metodología convencional de fijación en formol e inclusión en parafina del procesamiento por las técnicas histológicas de rutina.

\section{Resultados}

El resumen de los establecimientos evaluados por ecorregión, provincias y prevalencias se presentan en la Tabla 1.

Se detectó un establecimiento positivo en cada ecorregión, que equivale a un 3,8\% de seropositividad para Salta-Jujuy. No se hallaron casos positivos en la provincia de Jujuy mientras que la prevalencia en la provincia de Salta fue de $6,25 \%$. Los establecimientos positivos evaluados por ecorregión tuvieron valores de prevalencias de $2,5 \%$ en QA, $2,7 \%$ en CS y fue positivo el único establecimiento evaluado de VT, mientras que los resultados intra-majada fueron de 4,8\% QA, $10 \%$ CS y $55 \%$ VT. Estos datos están resumidos en la Tabla 2 .

Tanto en el caso de CS como en QA, no se apreciaron casos clínicos que hicieran sospechar de la presencia de la enfermedad y tampoco los propietarios manifestaron haberlo percibido. En el caso de VT, se trató de un tambo en el cual se tuvo la posibilidad de realizar un seguimiento del brote y donde el $48 \%$ de los animales tenía algún tipo de sinología clínica (Figura 1), el 46,8\% (51 animales) fueron casos de patología 
articular con los siguientes porcentajes: afecciones carpales $37,84 \%$ (42 animales), carpos e interfalángicas, $0,9 \%$ ( 1 animal), lesiones tarsales 3,6\% (4 animales) y coxofemoral solamente $0,9 \%$ ( 1 animal) que además tenía afectada la articulación atlantoodontoidea, ligamento y bolsas nucales. También se presentaron casos de mastitis y secado temprano cercano al parto $9 \%$ (10 animales) y se observó un caso de encefalitis en un cabrito de 50 días. Cabe destacar que varios animales presentaron más de una afección simultánea dificultándose la presentación del recuento frecuencial. Estos resultados se resumen en la figura 2.

Tabla 1. Establecimientos, eco-regiones, provincia, número de establecimientos positivos, número de establecimientos negativos, prevalencias por ecorregión e intra-majada (\%). $\mathrm{QA}=$ Quebradas áridas; $\mathrm{CS}=$ Chaco salteño; VT= Valles templados; Prev.= Seroprevalencia

\begin{tabular}{lccc}
\hline & QA & CS & VT \\
\hline Establecimientos & 40 & 37 & 1 \\
Salta & 10 & 37 & 1 \\
Jujuy & 30 & 0 & 0 \\
Positivos & 1 & 1 & 1 \\
Negativos & 39 & 36 & 0 \\
Prev. ecorregión & $2,5 \%$ & $2,7 \%$ & $100 \%$ \\
\hline Prev. Intra-majada & $4,8 \%$ & $10 \%$ & $55 \%$ \\
\hline
\end{tabular}

Tabla 2. Establecimientos positivo por ecorregión, animales seropositivos por establecimiento, total de animales evaluados por establecimiento, prevalencias intra-majada establecimiento/ecorregión (\%). QA= Quebradas áridas; $\mathrm{CS}=$ Chaco salteño; VT= Valles templados.

\begin{tabular}{|c|c|c|c|}
\hline & QA & CS & VT \\
\hline $\begin{array}{l}\text { Seropositivos por } \\
\text { establecimiento afectado }\end{array}$ & 1 & 2 & 11 \\
\hline $\begin{array}{l}\text { Total de animales } \\
\text { evaluados por } \\
\text { establecimiento }\end{array}$ & 21 & 20 & 20 \\
\hline Seroprev. intra-majada & $4,8 \%$ & $10 \%$ & $55 \%$ \\
\hline
\end{tabular}

En los casos que se logró realizar una necropsia, todos mostraron artritis que en sus inicios era de tipo serosa para después convertirse en proliferativa. En los más severos además se apreciaba necrosis caseosa (Figura 3). En animales de más larga evolución se observó erosión y adelgazamiento del cartílago articular que en algunos casos dejaba expuesto el hueso sub-condral (animal 2 y 5). En pulmón las lesiones fueron leves (animales 1, 4 y 5) y sólo en un animal se observaron lesiones macroscópicas (animal 4) caracterizadas por consolidación de los márgenes pulmonares. Los demás tejidos no presentaron lesiones macroscópicas de relevancia.

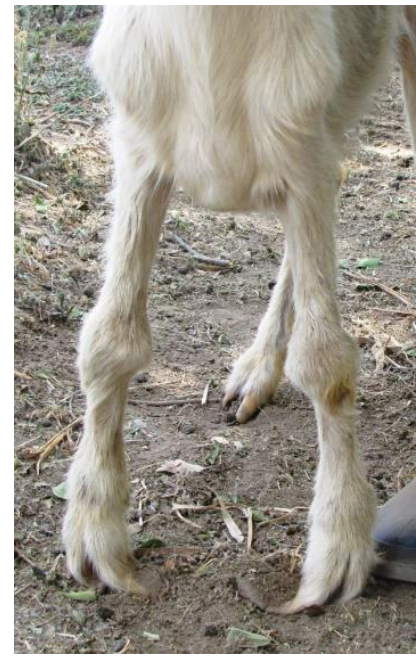

Figura 1. Artritis bilateral severa de los carpos en una cabrilla. Se observa deformación de ambos miembros.

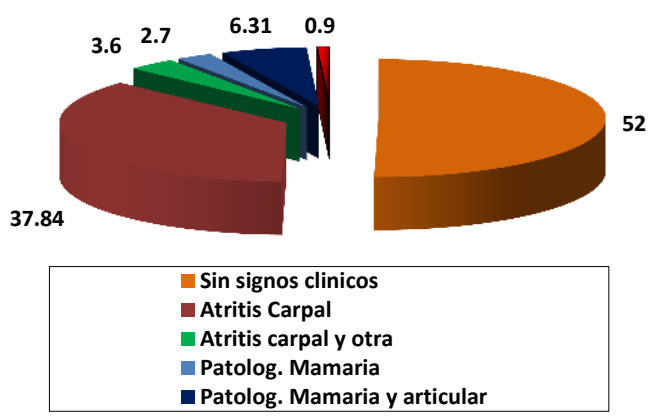

Figura 2. Porcentajes de signología clínica en el hato completo.

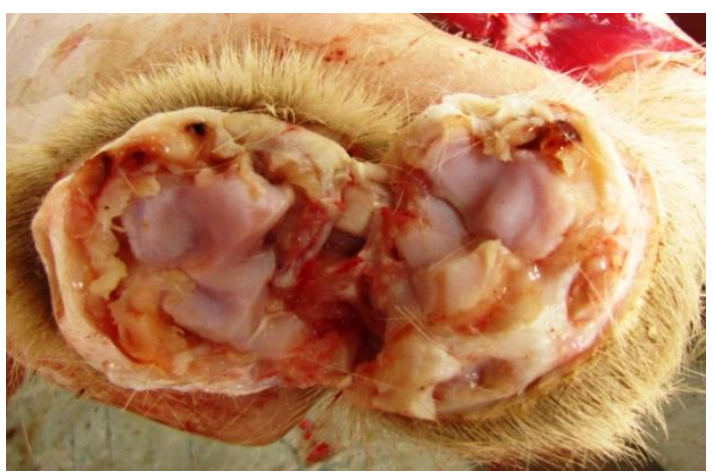

Figura 3. Lesión Macroscópica. Se observan lesiones proliferativas de la capsula articular que denota la cronicidad del cuadro.

Un resumen de los aspectos microscópicos de relevancia se presenta en las Tablas 3 y 4 . Microscópicamente las lesiones articulares se caracterizaron por la infiltración linfoidea, acompañada de proliferación papilar de capsula sinovial y fibroplasia del tejido conectivo sub-capsular (animal 1). En algunos casos 
muy severos se apreció necrosis coagulativa del tejido sinovial que quedaba alojada en el interior de la articulación (figura 4, A y B, animales 2, 3 y 5).

Tabla 3. Resumen de animales, edad y tejido afectado según el grado establecido (Ausente, leve, moderado y severo) de afección histopatológica encontrada.

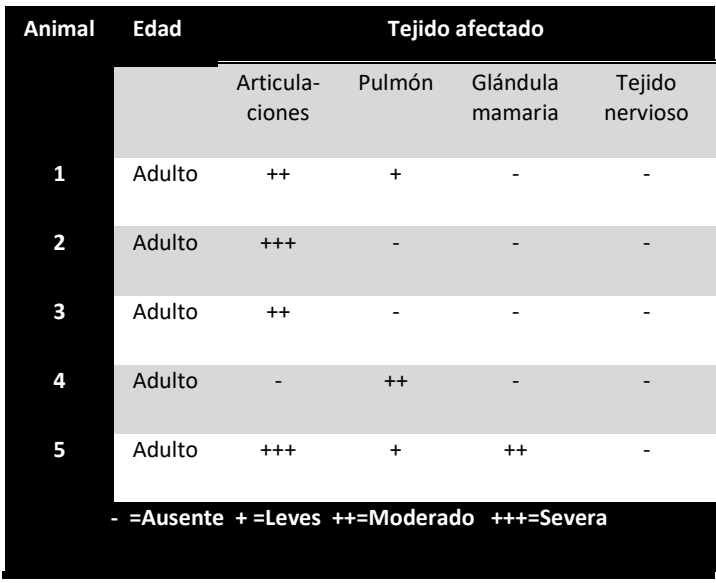

Tabla 4. Resumen de animales, tipo de lesiones histopatológicas articulares encontradas (infiltración, proliferación y necrosis) según el grado establecido (Ausente, leve, moderado y severo con presencia de clasificación distrófica).
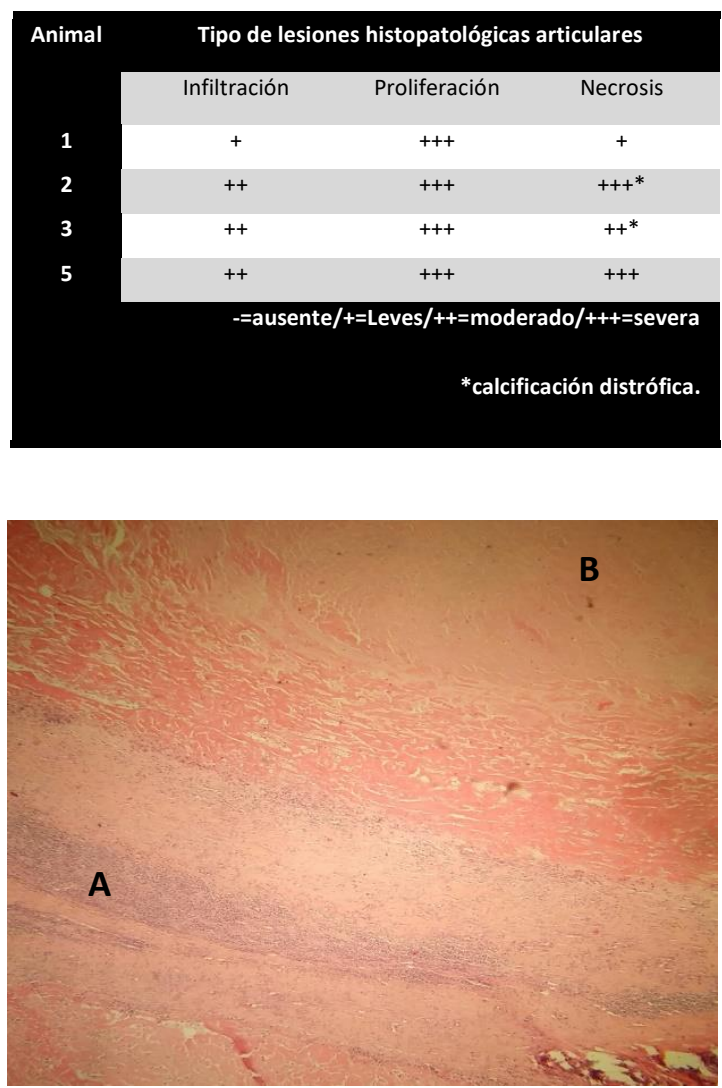

Figura 3. Lesión microscópica A- Proliferación de la cápsula articular con infiltración mononuclear. B- Necrosis.

\section{Discusión}

En la zona de QA de la provincia de Jujuy no se presentaron reactores en ningún establecimiento, mientras que si lo hubo en QA de Salta en una explotación familiar que poseía animales lecheros cruza Saanen con una prevalencia baja y sin signos clínicos. En el CS la prevalencia fue mayor con predominio de animales criollos y cruza Anglo Nubian, también dedicados a la producción láctea y sin lesiones visibles. El hecho de no encontrarse manifestaciones clínicas en estos dos establecimientos quedaría justificado por las bajas seroprevalencias, ya que muchos autores indican que los casos clínicos comienzan a visualizarse con prevalencias superiores al 20-30\% (Lujan et al, 2001, Tariba et al, 2015, Washburn. 2015, Lofstedt, 2016). Por el contrario, el caso positivo de VT de fue de alta prevalencia y con numerosas manifestaciones clínicas. Se produjo en un tambo comercial, con manejo semiintensivo de caprinos Saanen, el mismo tipo de establecimientos lecheros son los presentados por Rossanigo (2016a y 2016b) y Panei (2017). Esto podría indicar o bien una mayor susceptibilidad de las razas lecheras y/o una mayor facilidad de difusión de la enfermedad debidas al manejo productivo del tambo, sea comercial o de productores familiares. Otro motivo facilitador puede haber sido las importaciones en pie, de animales de tipo lechero que acontecieron en forma legal e ilegal durante los años 1995-2000 aproximadamente, sin ningún tipo de control sobre la enfermedad e incluso la importación de semen, de la cual se ha hecho uso y que es considerada actualmente como forma de contagio (Minguijon et al, 2015).

Los datos recolectados del seguimiento del caso en VT, muestran que la enfermedad es principalmente de curso articular, donde la articulación del carpo es la más frecuentemente afectada y a diferencia con reportes previos (Minguijon et al, 2015, Perez et al, 2015, Panei et al, 2017), las lesiones articulares presentan un alto grado de necrosis, dato particular que podría estar indicando diferencias en su grado de patogenicidad de la o las cepas circulantes, respecto a las conocidas. También cabe destacar, que la patología mamaria ocurrió principalmente en cabrillas de primer parto, cosa que podría afectar severamente su potencial génico y no dejar expresar fenotípicamente la real producción del animal, acarreando más perdidas subclínicas que se le sumen a las producidas por una baja en el consumo de materia seca debido al dolor articular.

Los resultados epidemiológicos contrastan con los presentados por Trezeguet (2013) en la provincia de Salta, con un $30 \%$ de predios positivos y prevalencias regionales del noroeste norte y sur de entre el 12 y $19 \%$, indicando que sería recomendable un cribado más exhaustivo para obtener resultados actualizados y representativos de la zona. 
En el país se ha descripto la presencia de casos clínicos con serología positiva, como en ovinos de Rio Negro 2001 (Robles et al, 2003), en caprinos del oeste pampeano en 2006 por Bedotti (2007), continuando por un caso de adenomatosis pulmonar ovina de Jujuy, que posteriormente fuera confirmada la presencia serológica del virus de CAEV asociado (Marin et al, 2012), junto al brote caprino de 2015 en San Luis donde los 9 animales evaluados con signología clínica fueron seropositivos con Checkit (Rossanigo et al, 2016a y 2016b; Álvarez et al, 2016) y finalmente el caso de Salta donde el $55 \%$ de los animales evaluados con Checkit $(11 / 20)$ se les confirma la presencia de anticuerpos contra la enfermedad (Dodero et al, 2016; Zabal et al, 2016). Estos casos trajeron aparejado un cambio en el estatus y la reglamentación sanitaria, exigiéndose actualmente la certificación serológica de negativo para la circulación y venta de reproductores mayores a 6 meses de edad (https://www.boletinoficial.gob.ar/\#!DetalleNorma/15 1302/20160923 27/10/2016). El diagnóstico posee grandes inconvenientes debido a los altos costos y baja disponibilidad del kit comercial. En febrero de 2017 se presenta el primer artículo sobre el aislamiento y caracterización de una cepa proveniente de caprinos de raza Saanen en la Provincia de Buenos Aires con sinología compatible con esta virosis (Panei et al, 2017). Toda esta información evidencia, más allá de los hallazgos serológicos conocidos, que la enfermedad está presente en Salta como también en el resto del país. Los casos clínicos descriptos son típicos, aunque la patogenicidad que implica la presencia de alto grado de necrosis incluyendo el tipo caseoso hace pensar en nuevas cepas que deben ser investigadas. EI brote cito en VT ha derivado una línea de investigación y se encuentra en las fases terminales del aislamiento viral, que nos permitirá comparar genéticamente las cepas presentes en el país - que no necesariamente puedan o deban tener el mismo origen - avanzando sobre la caracterización molecular y antigénica tan necesaria para un mayor conocimiento de este agente patógeno. Es fundamental el desarrollo de capacidades locales para el diagnóstico de la enfermedad y/o validación de técnicas diagnósticas precoces, sensibles, específicas como también asequibles para el mercado local, dado que es indispensable la detección de los animales positivos dentro del hato para poder llevar adelante cualquier tipo de medida de saneamiento que no fuera el rifle sanitario, aun así, es necesario el diagnóstico para la adquisición de nuevos animales libres de la enfermedad de manera inequívoca.

\section{Agradecimientos}

Se agradece la colaboración en la toma de muestras y acceso a los predios a la Ing. Agr. Marcela Martínez, a los Med. Vet. Sergio Cortez, German Grossberger, Cristina Rossetto, Juan D. Nievas, Alejandro Raia y al señor Antonio Salatín. Este trabajo ha sido financiado por el Instituto Nacional de Tecnología Agropecuaria (INTA).

\section{Bibliografía}

Álvarez I, Raia A, Porta N, Delgado F, Carosio A, Trono K, Rossanigo C. 2016. Evidencia preliminar del virus de la Artritis-encefalitis caprina (CAEV) y el virus Maedi-Visna (MVV) en cabras de San Luis. V11. XXI Reunión científico técnica de la AAVLD. San Salvador de Jujuy.

Bedotti DO, Fort MC, Giménez H, Langhoff A, Garré J, Hertsommer O. 2007. Descripción de un caso de ArtritisEncefalitis caprina en la provincia de La Pampa, Argentina. Pp: 163-165.V Congreso de Especialistas en pequeños rumiantes y camélidos sudamericanos, Mendoza, Argentina.

De Souza TS, Pinheiro RR, Costa JN, De Lima CCV, Andrioli A, De Azevedo DAA, Dos Santos VWS, Araújo JF, De Sousa ALM, Pinheiro DNS, Fernandes FMC, Costa Neto AO. 2015. Interspecific transmission of small ruminant lentiviruses from goats to sheep. Braz. J. Microbiol. 46: 867-874.

Dodero AM., Suarez VH, Bertoni EA., Rossetto EA, Martinez GM, Romera SA, Quiroga Roger J, Brihuega V, Raia NA, Viñabal $A E$, Salatin AO. 2015. Enfermedades en hatos caprinos de valles áridos y chaco semiárido. IX Seminario de la Fundación "Charles Louis Davis". Salta, Argentina 2015.

Dodero AM, Suarez VH, Salatin AO, Raia NA, Pinto GB. 2016. Artritis y Encefalitis caprina en las provincias de Salta y Jujuy. V7. XXI Reunión científico técnica de la AAVLD. San Salvador de Jujuy.

Jolly PE, Narayan O. 1989. Evidence for interference, coinfections, and intertypic virus enhancement of infection by ovine-caprine lentiviruses. J Virol. 63: 4682-4688.

Jones TC, Hunt RD, King NW. 1997. Diseases caused by viruses. (pp:330-338) En: Lippincott Willams and Willams (eds.) Veterinary Pathology. 6th.edition. Blackwell Publishing, Baltimore.

Lofstedt, J. 2016. Merck veterinary manual. Ed. S. E. Aiello. Kenilwork, NJ. 747-749 pp.

Luján L, Juste RA, Berriatua E, Badiola JJ. 2001.epidemiología y control. El virus Maedi-Visna en España. Ovis 72: 1-13

Minguijón E, Reina R, Pérez $M$, Polledo L, Villoria $M$, Ramírez H, Leginagoikoa I, Badiola JJ, García-Marín JF, De Andrés D, Luján L, Amorena B, Just RA. 2015. Small ruminant lentivirus infections and diseases. Vet. Microbiol. 181: 75-89.

Marín RE, Setti W, Carrillo BJ y Wooton S. 2012. Descripción de un caso de Adenomatosis Pulmonar Ovina en la Puna de Jujuy, Argentina. Vet. Arg. 29: 1-5.

Narayan O, Clements JE, Strandberg JD. 1980. Biological characterization of the virus causing leukoencephalitis and arthritis in goats. J. Gen. Virol. 50: 69-79.

Panei JC, Gos ML, Valera AL, Galosi CM y Echeverria MG. 2017. First isolation and nucleotide comparison of the gag gene of the caprine arthritis encephalitis virus circulating in naturally infected goats from Argentina. Open Vet. J. 7: 32-35

Pepin M, Vitu C, Russo P, Mornex JF, Peterhans E. 1998. Maedi-Visna virus infection in sheep: a review. Vet. Res. 29: 341-367. 
Perez M, Biescas E, Reina R, Glaria I, Marin B, Marquina A, Salazar E, Lvarez NA, De Andre'S D, Fantova E, Badiola J J, Amorena B y Lujan L. 2015. Small Ruminant LentivirusInduced Arthritis: Clinicopathologic Findings in Sheep Infected by a Highly Replicative SRLV B2 Genotype. Vet. Path. 52: 132139.

Radostits OM, Gay CC, Blood DC, Hinchcliff KW. 2002. Medicina Veterinaria. Tratado de las enfermedades del ganado bovino, ovino, porcino, caprino y equino. Ed. McGraw-Hill-Interamericana. Madrid. 1451 pp.

Robles, C, Uzal, F. 1991. Guía práctica de necropsia en ovinos y caprinos. Ed. Hemisferio Sur S.A. Buenos Aires. 20 pp.

Robles CA, Layana JA, Cabrera RF, Raffo F, Cutlip R. 2003. Estudio serológico retrospectivo de Maedi (Neumonía Progresiva) en ovinos y de Artritis-Encefalitis en caprinos de Patagonia, Argentina. Revista de Medicina Veterinaria. 84: 96-99.

Rossanigo C, Delgado G, Raia A, Carosio A, Alvares I, Rey J, Rodriguez M, Paje W. 2016a. Presentacion clinica de un caso de artritis-encefalitis caprina (CAE) y toxoplasmosis en San Luis (Argentina). V12. XXI Reunión científico técnica de la AAVLD. San Salvador de Jujuy.

Rossanigo C, Delgado C, Carosio A, Raia A, Alvares I, Rodriguez M, Paje W, Pinto G. 2016b. Reporte de un caso de artritis-encefalitis caprina en la provincia de San Luis. Boletín no 3 PNSA. Ed. INTA.

Smith MC, Sherman DM. 1994. Musculoskeletal System (pp. 73-82) En: Liippincot Willams and Wiillamns. (eds.) Goat Medicine. Baltimore

Rowe JD, East NE. 1997. Risk factors for transmission and methods fro control of caprine arthritis-encephalitis virus infection. Vet. Clin. North. America: Food Anim. Pract. 13: 3553

Tariba B, Kostelić A, Salamon D, Roić B, Benić M, Prvanović Babić N, Salajpal K. 2015. Prevalence of caprine arthritis encephalitis virus in association with clinical arthritis in six production farms of French Alpine goats in north-western Croatia. Poljoprivreda 21: 135-137.

Trezeguet MA; Suarez R, Barral M, Periolo L, Maidana F, Farías CE, Rodríguez P C,.; Debenedetti CE, Marcos RT Y Cosentino B. 2013. Situación epidemiológica de maedi-visna y artritis encefalitis caprina en la Argentina. SNS. 1: 2314-2901.

Washburn K. 2015. Large Animal Internal Medicine. Fifth edition. Ed. Bradford P. Smith, Missouri. 1100-1102 pp

Zabal O, Dodero AM, Reina R, Valenzano M Y Peralta AV. 2016. XXI Obtención y evaluación de cultivos primarios de origen epitelial para el aislamiento de los virus CAEV y ORFV. V1. Reunión científico técnica de la AAVLD. San Salvador de Jujuy.

Zink MC, Johnson LK. 1994. Pathobiology of lentivirus infections of sheep and goats. Vir. Res. 32:139-154. 